\title{
Whole-exome sequencing of duodenal neuroendocrine tumors in patients with neurofibromatosis type 1
}

\author{
Michaël Noë $\mathbb{D}^{1,2} \cdot$ Antonio Pea ${ }^{3,4} \cdot$ Claudio Luchini $\mathbb{D}^{1,5} \cdot$ Matthäus Felsenstein ${ }^{1} \cdot$ Stefano Barbi $\mathbb{D}^{5} \cdot$ Feriyl Bhaijee ${ }^{1}$. \\ Raluca Yonescu ${ }^{1}$ - Yi Ning ${ }^{1}$ - N. Volkan Adsay ${ }^{6}$. Giuseppe Zamboni ${ }^{5,7} \cdot$ Rita T. Lawlor ${ }^{8}$. Aldo Scarpa ${ }^{5,8}$. \\ G. Johan A. Offerhaus ${ }^{2} \cdot$ Lodewijk A. A. Brosens $^{1,2,9} \cdot$ Ralph H. Hruban $^{1,10} \cdot$ Nicholas J. Roberts $^{1,10} \cdot$ Laura D. Wood $^{1,10}$
}

Received: 30 January 2018 / Revised: 30 April 2018 / Accepted: 3 May 2018 / Published online: 30 May 2018

(c) United States \& Canadian Academy of Pathology 2018

\begin{abstract}
Neurofibromatosis type 1 (NF1) is a hereditary cancer predisposition syndrome characterized by frequent cutaneous and nervous system abnormalities. Patients with NF1 also have an increased prevalence of multiple gastrointestinal and peripancreatic neoplasms-neuroendocrine tumors of the ampulla that express somatostatin are particularly characteristic of NF1. In this study, we characterize the genetic alterations of a clinically well-characterized cohort of six NF1-associated duodenal neuroendocrine tumors using whole-exome sequencing. We identified inactivating somatic mutations in the NFI gene in three of six tumors; the only other gene altered in more than one tumor was IFNB1. Copy number analysis revealed deletion/loss of heterozygosity of chromosome 22 in three of six patients. Analysis of germline variants revealed germline deleterious NF1 variants in four of six patients, as well as deleterious variants in other tumor suppressor genes in two of four patients with deleterious NF1 variants. Taken together, these data confirm the importance of somatic inactivation of the wildtype NF1 allele in the formation of NF1-associated duodenal neuroendocrine tumors and suggest that loss of chromosome 22 is important in at least a subset of cases. However, we did not identify any genes altered in the majority of NF1-associated duodenal neuroendocrine tumors that uniquely characterize the genomic landscape of this tumor. Still, the genetic alterations in these tumors are distinct from sporadic neuroendocrine tumors occurring at these sites, highlighting that unique genetic alterations drive syndromic tumors.
\end{abstract}

These authors contributed equally: Michaël Noë, Antonio Pea.

Electronic supplementary material The online version of this article (https://doi.org/10.1038/s41379-018-0082-y) contains supplementary material, which is available to authorized users.

$\checkmark$ Laura D. Wood

ldwood@jhmi.edu

1 Department of Pathology, Sol Goldman Pancreatic Cancer Research Center, The Johns Hopkins University School of Medicine, Baltimore, MD, USA

2 Department of Pathology, University Medical Center Utrecht, Utrecht, The Netherlands

3 Department of Surgery, Sol Goldman Pancreatic Cancer Research Center, The Johns Hopkins University School of Medicine, Baltimore, MD, USA

4 Department of Surgery, University and Hospital Trust of Verona, Verona, Italy

\section{Introduction}

Hereditary cancer predisposition syndromes, in which germline alterations lead to an increased risk of neoplasia, represent a unique opportunity to study tumorigenesis, and the results of these studies can have important clinical implications.

5 Department of Diagnostics and Public Health, Section of Pathology, University of Verona, Verona, Italy

6 Medical College of Wisconsin, Milwaukee, WI, USA

7 Sacro Cuore Don Calabria Hospital, 37024 Negrar, Verona, Italy

8 ARC-Net Research Center, University of Verona, Verona, Italy

9 Department of Pathology, Radboud University Medical Center, PO Box 9101, 6500 HB Nijmegen, The Netherlands

10 Department of Oncology, Sol Goldman Pancreatic Cancer Research Center, The Johns Hopkins University School of Medicine, Baltimore, MD, USA 
Neurofibromatosis type 1 (NF1), also known as von Recklinghausen disease, is an autosomal dominant hereditary cancer predisposition syndrome caused by inactivating germline alterations in the $N F 1$ gene encoding neurofibromin on chromosome 17q [1]. This syndrome affects $\sim 1$ in 3000 individuals and is characterized by a constellation of clinical findings, including café-au-lait spots, axillary freckling, optic gliomas, and neurofibromas, a small proportion of which can transform into malignant peripheral nerve sheath tumors [1].

Although less common than the cutaneous and nervous system abnormalities, several gastrointestinal and peripancreatic neoplasms also occur at increased prevalence in patients with NF1, including gastrointestinal stromal tumors and neuroendocrine tumors of the duodenum and ampulla [2]. Intriguingly, the majority of these neuroendocrine tumors typically have unique morphological features, including prominent psammomatous calcifications, and express the hormone somatostatin [2]. Previous genomic analysis of NF1-associated malignant peripheral nerve sheath tumors has revealed critical drivers of malignant transformation in the SUZ12 gene in these tumors [3]. However, little is known about the genetic alterations that drive the formation of gastrointestinal and peripancreatic tumors in NF1 patients.

Multiple lines of evidence suggest that functional and syndromic neuroendocrine tumors will harbor genomic alterations distinct from those in sporadic non-functional neuroendocrine tumors that have previously been characterized in depth [4-6]. Whole-exome sequencing of a specific clinicopathological group of pancreatic neuroendocrine tumors, sporadic insulinomas, revealed a unique frequently altered hotpsot mutation, suggesting that specific clinical and pathological categories of neuroendocrine tumors are driven by distinct genetic alterations [7]. In addition, previous studies have demonstrated that gastrointestinal stromal tumors in NF1 patients are genetically distinct from sporadic gastrointestinal stromal tumors [8]. As such, we hypothesize that NF1-associated duodenal neuroendocrine tumors may be a genetically unique category of neuroendocrine tumors. In order to characterize the genomic landscape of these neoplasms in NF1 patients, we performed whole-exome sequencing on a clinically wellcharacterized cohort of six neoplasms.

\section{Materials and methods}

This study (including the analysis of germline DNA) was approved by the Institutional Review Boards of The Johns Hopkins Hospital and Emory University Hospital. The surgical pathology databases of The Johns Hopkins Hospital and Emory University Hospital were searched for surgically resected neuroendocrine tumors of the ampulla with psammomatous calcifications or immunohistochemical reactivity for somatostatin in patients clinically diagnosed NF1. From tumor samples SOM3-6, formalin-fixed paraffin-embedded tissue blocks of tumor and matched normal were reviewed by a pathologist and macrodissected to enhance neoplastic cellularity. Cores of neoplastic and normal tissue were isolated from formalin-fixed paraffinembedded blocks using a $0.6 \mathrm{~mm}$ needle. For tumor samples SOM7-8, fresh-frozen tumor and normal (harvested at the time of surgery and subsequently banked at $-70{ }^{\circ} \mathrm{C}$ ) were reviewed by a pathologist and macrodissected after frozen section analysis to enhance neoplastic cellularity. DNA was extracted using the QIAamp DNA FFPE tissue kit (Qiagen) or QIAamp DNA mini kit (Qiagen) according to the manufacturer's instructions and quantified using the Qubit 2.0 (Thermo Fisher).

Whole-exome sequencing was performed at Personal Genome Diagnostics with capture using the Agilent SureSelect paired end version 4.0 library preparation followed by sequencing on an Illumina HiSeq, as previously described [9]. Somatic mutations were identified using the VariantDx pipeline as previously described [9]. In order to identify copy number alterations, we utilized the ASCAT software [10]. Specifically, we identified $\sim 40,000$ singlenucleotide polymorphisms in the target region with high global minor allele frequency-the normalized number of reads along with the estimated B allele frequency was then used as input for ASCAT. However, owing to high dispersion in local sequencing coverage, most of the samples could not be reliably modeled with ASCAT. Therefore, the plots representing normalized read depth and B allele frequency along the chromosomes were visually inspected to identify large chromosomal alterations based on lower number of reads and absence of the band corresponding to heterozygous single-nucleotide polymorphisms in the B allele frequency plot. In addition, we performed complimentary copy number analysis with CNVkit to utilize both the on-target and off-target reads [11]. Scatterplots were created with CNVkit and visually inspected for each chromosome, and copy number status of tumors were compared to the matched normal sample from the same patient. Deleterious germline variants were identified after alignment of sequence read files to the human genome (hg19) with Burrows-Wheeler Aligner and variant calling with Varscan 2 [12, 13]. Classification of variants as either benign, of unknown significance, or deleterious was performed as previously described [14].

Fluorescence in situ hybridization was performed on formalin-fixed paraffin-embedded specimens using a dual color probe (Abbott Molecular). The probe is designed for the detection of chromosomal region $9 \mathrm{q} 34.12$ harboring the $A B L 1$ gene (orange fluorochrome) and the chromosomal 
region 22q11.23, harboring the $B C R$ gene (green fluorochrome). Prior to hybridization, formalin-fixed paraffinembedded slides were deparaffinized using a VP2000 processor (Abbott Molecular). The slides and the probe were co-denatured at $75^{\circ} \mathrm{C}$ for 5 minutes and allowed to anneal over night at $37^{\circ} \mathrm{C}$, in humidified atmosphere. Following incubation the slides were washed in $2 \times$ saline-sodium citrate (SSC)/ $/ 0.3 \% \mathrm{NP}-40$ for 2 minutes at $72{ }^{\circ} \mathrm{C}$, and for 2 minutes at room temperature in $2 \times \mathrm{SSC}$. The slides were counterstained with 4',6-diamidino-2-phenylindole and a cover slip was applied using Vectashield mounting medium (H-1000, Vector Laboratories, Inc.). A fluorescence microscope was used to evaluate signal patterns generated by each probe. In normal interphase cells, the signal pattern showed two orange signals (two copies of $A B L 1$ gene) and two green signals (two copies of $B C R$ gene). Samples showing $>10 \%$ of the 50 analyzed cells with less than or more than two signals are interpreted as loss or gain, respectively, for the targeted chromosome locus.

\section{Results}

\section{Clinical and pathological data}

We identified six duodenal neuroendocrine tumors occurring in patients who were clinically diagnosed with NF1. Microscopic evaluation of these tumors showed the typical morphological pattern of homogeneous neoplastic cells with abundant clear or granular eosinophilic cytoplasm and round, smooth nuclei with finely stippled chromatic. The neoplastic cells were organized in nests, trabeculae, and acinar structures, often with prominent interspersed psammomatous calcifications (Fig. 1). Clinical and pathological features are summarized in Table 1. All the tumors originated in the duodenum or around the major or minor duodenal papilla. The mean age at time of resection was 52 (range 40-61), and $67 \%$ of the patients were male. The mean size of the primary tumor was $2.6 \mathrm{~cm}$, and $50 \%$ had lymph node metastases at the time of surgery. When performed, Ki67 proliferation rate was $<2 \%$ in all cases, consistent with WHO Grade 1 based on current classifications $[15,16]$. Immunolabeling for somatostatin was positive in four cases, and none had immunolabeling for other commonly expressed pancreatic hormones such as insulin and glucagon. One case was negative for somatostatin labeling, but this case had characteristic glandular psammomatous morphology, as did another tumor in which somatostatin immunohistochemistry was not available. All patients had other histological features suggestive of NF1, including duodenal neurofibromas, duodenal gastrointestinal stromal tumor, and neurofibromatosis-associated vasculopathy [17].
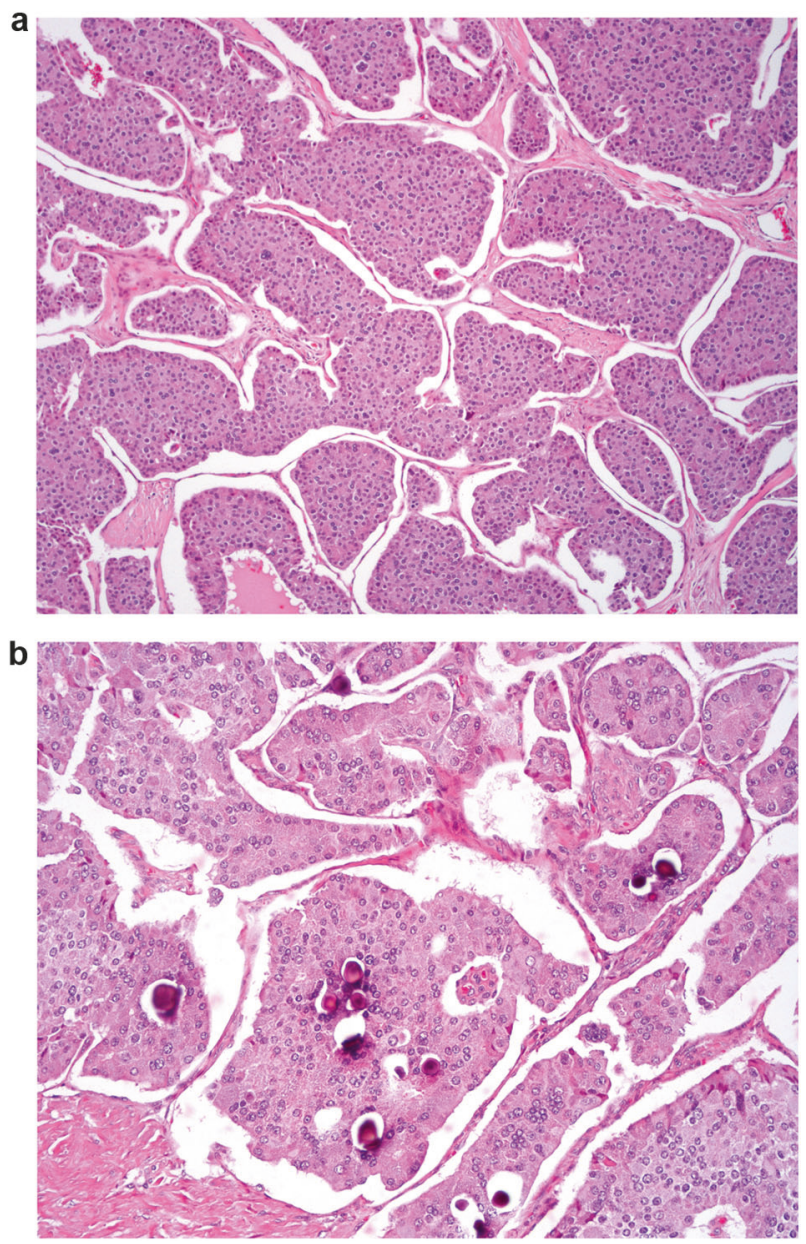

Fig. 1 Histopathology of duodenal neuroendocrine tumors in NF1 patients. a A low-power view of duodenal neuroendocrine tumor shows characteristic nested architecture. Hematoxylin and eosin, $\times 10$. b A high-power view shows characteristic cytologic features, including monotonous round nuclei with finely stippled chromatin, amphophilic granular cytoplasm, and psammomatous calcifications. Hematoxylin and eosin, $\times 20$

\section{Whole-exome sequencing}

Whole-exome sequencing of six NF1-associated duodenal neuroendocrine tumors revealed a range of 2-11 nonsynonymous somatic mutations per tumor (Table 2, Supplementary Table 1, Supplementary Table 2). Only two genes were mutated in more than one tumor: inactivating mutations (two frameshift and one nonsense) in $N F 1$ occurred in three tumors, and missense mutations in IFNBI occurred in two tumors. In addition, somatic mutation in the oncogenic hotspot in codon 600 in $B R A F$ was identified in a single tumor.

We also utilized the whole-exome sequencing data to identify copy number alterations using two complimentary approaches-ASCAT [10], which analyzed the B allele frequency of germline single-nucleotide polymorphisms in the target region, and CNVkit [11], which analyzed copy number based on read depth in the on-target and off-target 


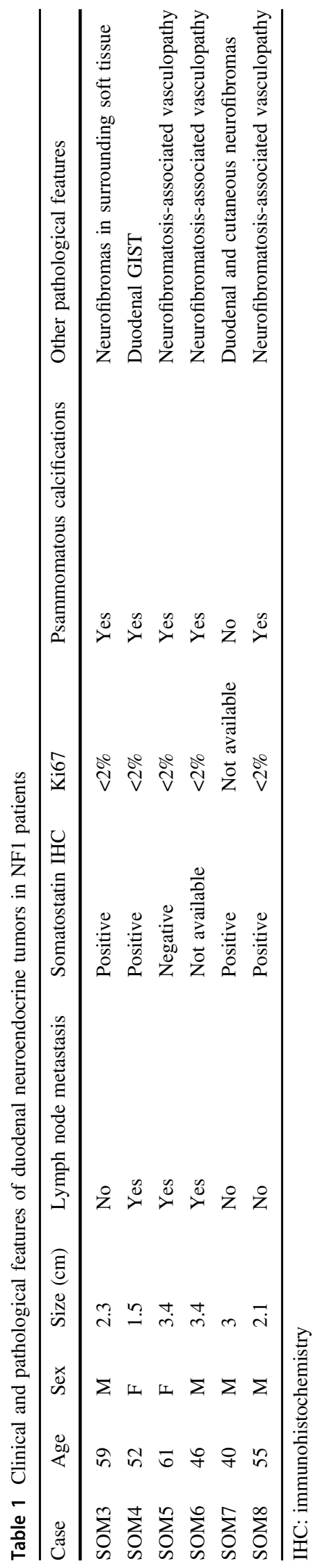

Table 2 Somatic mutations identified by whole-exome sequencing in NF1-associated duodenal neuroendocrine tumors

\begin{tabular}{|c|c|c|c|}
\hline Tumor & Gene symbol & Mutation type & $\begin{array}{l}\text { Amino-acid } \\
\text { change }\end{array}$ \\
\hline SOM3 & $B R A F$ & Nonsynonymous coding & p.V600E \\
\hline SOM3 & CEACAM2O & Nonsynonymous coding & p.R332W \\
\hline SOM3 & DCTN1 & Nonsynonymous coding & p.R997L \\
\hline SOM3 & FAM135B & Nonsynonymous coding & p.S105N \\
\hline SOM3 & LRRC39 & Frameshift & p.T240fs \\
\hline SOM3 & $M S N$ & In-frame deletion & p.344delE \\
\hline SOM3 & MTRNR2L2 & Nonsynonymous coding & p.S12L \\
\hline SOM3 & $N E B$ & Nonsynonymous coding & p.L560V \\
\hline SOM3 & NELL2 & Nonsynonymous coding & p.G841V \\
\hline SOM3 & $N F 1$ & Frameshift & p.F150fs \\
\hline SOM3 & USP34 & Nonsynonymous coding & p.V609L \\
\hline SOM4 & $P C N T$ & Nonsynonymous coding & p.G136S \\
\hline SOM4 & $S M C 3$ & Splice site acceptor & splice site \\
\hline SOM5 & CAMKK2 & Nonsynonymous coding & p.R104Q \\
\hline SOM5 & $D A C T 1$ & Nonsynonymous coding & p.S759L \\
\hline SOM5 & $J A K M I P 1$ & Nonsynonymous coding & p.G108D \\
\hline SOM5 & PPIP5K1 & Nonsynonymous coding & p.F1144V \\
\hline SOM5 & RNASE7 & Nonsynonymous coding & p.P48T \\
\hline SOM5 & SP4 & Nonsynonymous coding & p.K705N \\
\hline SOM6 & AIMIL & Nonsynonymous coding & p.R25W \\
\hline SOM6 & ANO10 & Frameshift & p.D45fs \\
\hline SOM6 & ATXN10 & Nonsynonymous coding & p.E312K \\
\hline SOM6 & FETUB & Frameshift & p.I147fs \\
\hline SOM6 & $F G A$ & Splice site acceptor & splice site \\
\hline SOM6 & IFNBI & Nonsynonymous coding & p.A76S \\
\hline SOM6 & $R D M 1$ & Nonsynonymous coding & p.R143K \\
\hline SOM6 & $V W A 8$ & Nonsynonymous coding & p.I709K \\
\hline SOM6 & WBSCR17 & Nonsynonymous coding & p.G314R \\
\hline SOM7 & $\mathrm{CDH} 23$ & Nonsynonymous coding & p.G540S \\
\hline SOM7 & $E Z H 2$ & Nonsynonymous coding & p.D185H \\
\hline SOM7 & IFNBI & Nonsynonymous coding & p.G135R \\
\hline SOM7 & $M Y O 1 B$ & Nonsynonymous coding & p.M493L \\
\hline SOM7 & $N F 1$ & Frameshift & p.W267fs \\
\hline SOM8 & AHCYL2 & Nonsynonymous coding & p.R468H \\
\hline SOM8 & IL5 & Nonsynonymous coding & p.V17M \\
\hline SOM8 & $N F 1$ & Nonsense & p.Q1822X \\
\hline SOM8 & $P L S 3$ & Nonsynonymous coding & p.K300N \\
\hline
\end{tabular}

See Supplementary Table 2 for complete data on all identified somatic mutations

reads. Both of these approaches revealed deletion/loss of heterozygosity of chromosome 22 in three tumors (Supplementary Figure 1). Key somatic alterations in each tumor are summarized in Table 3.

In addition to the identification of somatic genetic events in NF1-associated duodenal neuroendocrine tumors, we also analyzed the germline sequencing data for deleterious 
Table 3 Summary of key genetic alterations in NF1-associated duodenal neuroendocrine tumors

\begin{tabular}{llll}
\hline Tumor & $\begin{array}{l}\text { Somatic NF1 } \\
\text { mutation }\end{array}$ & $\begin{array}{l}\text { Germline NF1 } \\
\text { variant }\end{array}$ & $\begin{array}{l}\text { Chromosome 22 } \\
\text { copy number }\end{array}$ \\
\hline SOM3 & p.F150fs & None identified & Deletion/LOH \\
SOM4 & None identified & p.S436X & Wild-type \\
SOM5 & None identified & p.Q514fs & Deletion/LOH \\
SOM6 & None identified & None identified & Deletion/LOH \\
SOM7 & p.W267fs & p.T1951fs & Wild-type \\
SOM8 & p.Q1822X & p.L1152fs & Wild-type \\
\hline
\end{tabular}

Table 4 Deleterious germline variants identified in NF1 patients with duodenal neuroendocrine tumors

\begin{tabular}{llll}
\hline Patient & Gene & Genomic DNA alteration & Amino-acid alteration \\
\hline SOM4 & NF1 & chr17:29533304 C>A & p.S436X \\
SOM5 & TSC1 & chr9:135773001insA & Splice site \\
SOM5 & NF1 & chr17:29546036delAG & p.Q514fs \\
SOM7 & NF1 & chr17:29661957insA & p.T1951fs \\
SOM8 & FANCC & chr9:98011507delC & p.D23fs \\
SOM8 & NF1 & chr17:29559859delACTC & p.L1152fs \\
\hline
\end{tabular}

variants in human cancer genes (Table 4). We identified three frameshift germline variants and one nonsense germline variant in $N F 1$; four patients had deleterious germline variants in NFl. In addition, two of these four patients also had deleterious germline variants in other known cancer genes, including a frameshift deletion in FANCC and a splice site mutation in TSCl, each in one patient.

\section{Fluorescence in situ hybridization}

In order to confirm the result of our copy number analysis utilizing whole-exome sequencing data, we performed fluorescence in situ hybridization for chromosomes 9 and 22 on neoplastic formalin-fixed paraffin-embedded tissue from all six tumors. We identified loss of chromosome 22 by fluorescence in situ hybridization in the three tumors with whole-exome sequencing data suggestive of deletion/ loss of heterozygosity, whereas the other three tumors retained two copies of both chromosomes 9 and 22 (Fig. 2).

\section{Discussion}

We report whole-exome sequencing of a clinically wellcharacterized cohort of duodenal neuroendocrine tumors arising in patients with NF1. In the literature, these tumors are often described as 'somatostatinomas' owing to their characteristic expression of somatostatin by immunohistochemistry, though clinical symptoms caused by somatostatin expression are exceedingly rare. Other features often seen in
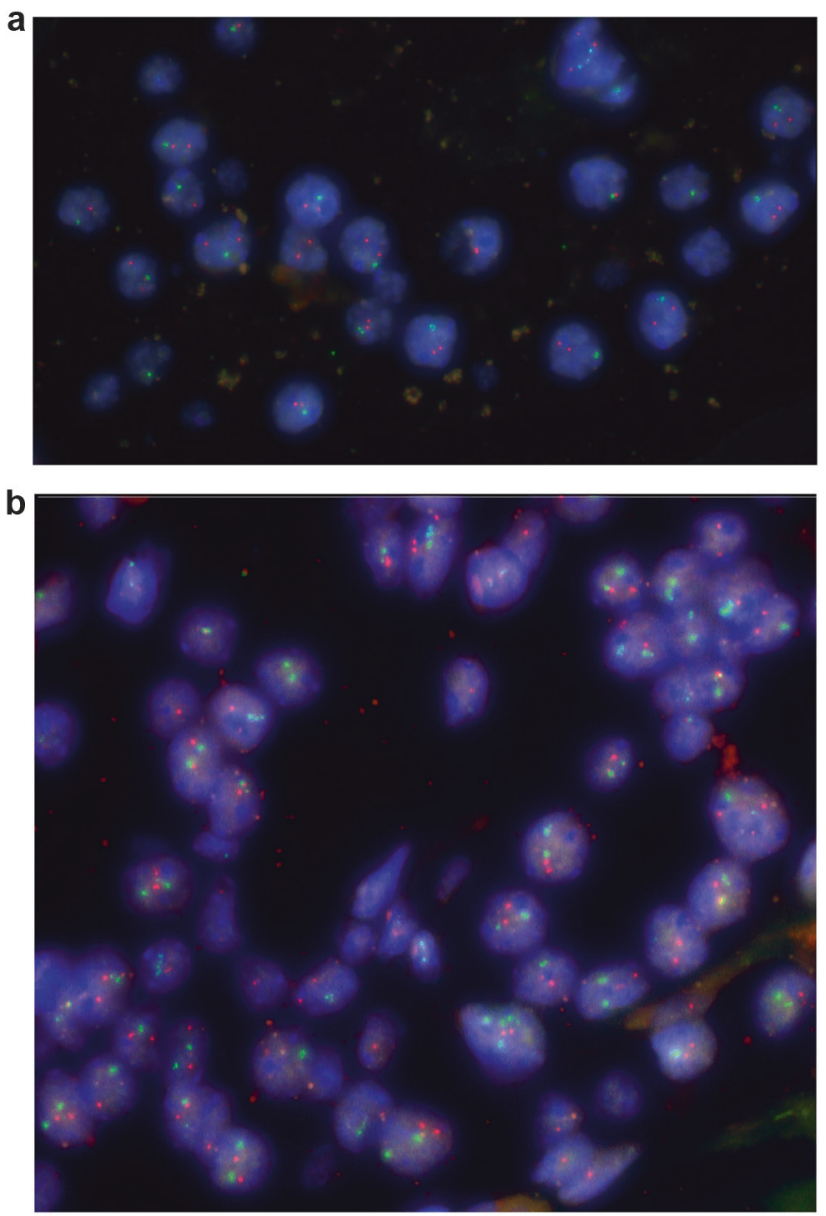

Fig. 2 Fluorescence in situ hybridization of chromosomes 9 and 22 in NF1-associated duodenal neuroendocrine tumors. a Fluorescence in situ hybridization demonstrates loss of chromosome 22 in neoplastic cells, as they contain two red signals (chromosome 9) but only one green signal (chromosome 22). b Fluorescence in situ hybridization on a tumor without chromosome 22 deletion shows two signals in both the red and green probes

neuroendocrine tumors in patients with NF1 are glandular morphology and presence of psammoma bodies. There is substantial overlap between neuroendocrine tumors with somatostatin expression, glandular neuroendocrine tumors with psammoma bodies, and neuroendocrine tumors associated with NF1 [1, 18]. As such, these lesions have been previously called "ampullary somatostatinoma" and "glandular duodenal neuroendocrine tumor". However, in this study, we use the general term "duodenal neuroendocrine tumor", as not all analyzed lesions expressed somatostatin or showed glandular morphology. The expression of other pancreatic hormones, including gastrin and serotonin, was not analyzed in this study though could be of interest for future studies describing these lesions at the protein level. Of note, sporadic somatostatinomas can show focal expression for gastrin or serotonin [19].

The most common intragenic somatic alteration in this tumor type was inactivating mutation in NF1 (either by frameshift or nonsense mutation), which occurred in three 
tumors. In addition to these small somatic mutations, we also identified somatic deletion/loss of heterozygosity of chromosome 22 in three tumors in our whole-exome sequencing data and confirmed these deletions by fluorescence in situ hybridization. Because the deletion includes the entire chromosome, it is not possible to identify the targeted driver gene. However, multiple well-characterized tumor suppressor genes are located on chromosome 22, including $N F 2$ and SMARCB1, which lead to neurofibromatosis type 2 and schwannomatosis, respectively [20-22]. Still, the lack of somatic mutations in these tumor suppressor genes raises the alternative possibility that other types of alterations, such as epigenetic modifications or alterations in non-coding regions, may complement the deletion/loss of heterozygosity of chromosome 22 .

Overall, the somatic mutations identified in duodenal neuroendocrine tumors in NF1 patients are unique from those identified in sporadic pancreatic neuroendocrine tumors well as sporadic small intestinal neuroendocrine tumors [4-6]mutations in $N F I$ and IFNBI do not occur commonly in these other tumor types, nor does loss of chromosome 22. In addition, somatic mutations in genes frequently altered in pancreatic neuroendocrine tumors (MENI, ATRX, DAXX) and small intestinal neuroendocrine tumors $(C D K N 1 B)$ were absent in our cohort. However, to our knowledge, no study has specifically analyzed sporadic duodenal neuroendocrine tumors, which would be the most appropriate comparison for our lesions. Analysis of these sporadic lesions, with subsequent comparison with the genetic alterations in syndromic lesions, remains a future direction.

The genomic landscapes of NF1-associated neoplasms reported in the literature to date is variable. The mutations in the duodenal neuroendocrine tumors in our cohort are distinct from those previously reported in malignant peripheral nerve sheath tumors, which is one of most comprehensively characterized NF1-associated neoplasms. Although the somatic "second hit" in NFl has been reported in NF1-associated malignant peripheral nerve sheath tumors, our tumors lacked mutations in SUZ12, EED, and CDKN2A that frequently occur in this tumor type [3, 23]. However, deletions of IFNBI (the only gene other than $N F 1$ with somatic mutations in more than one tumor in our cohort) have been reported in $20 \%$ of malignant peripheral nerve sheath tumors, suggesting perhaps a common genetic driver among NF1-associated neoplasms [24]. Overall, the alterations in NF1-associated duodenal neuroendocrine tumors are more similar to those reported in plexiform neurofibromas in NF1 patients-whole-exome sequencing has previously revealed only a small number of somatic mutations in these benign NF1-associated neoplasms, with somatic mutations in $\mathrm{NFl}$ as the most common alteration [25]. Similarly, whole-genome sequencing analysis of a small number of NF1-associated pilocytic astrocytomas revealed that somatic inactivation of $N F 1$ was the only recurrent genetic alteration [26]. Of note, loss of chromosome 22 has not been previously reported in NF1-associated neoplasms.

In three of the six tumors, we did not identify somatic NF1 mutations. In these cases, deletion/loss of heterozygosity of the wild-type NFI allele may not have been accurately identified by our approach. Although our copy number analysis could robustly identify arm-level chromosomal alterations (such as deletion/loss of heterozygosity of chromosome 22), it is possible that our analysis missed focal deletions of the wild-type copy of the gene in these cases due to sequencing approach and coverage depth. Such focal deletions have been previously reported as a mechanism for somatic NF1 inactivation [3]. Alternatively, methylation has been reported as an alternative mechanism for inactivation of the second NFI allele, which would not have been identified by our whole-exome sequencing approach [26]. Although immunohistochemical detection of the neurofibromin protein encoded by $N F 1$ is another possible assay to interrogate this gene, immunohistochemistry has not been shown to be a reliable predictor of genetic and epigenetic alterations in $\mathrm{NFI}$ $[27,28]$. Thus, we did not use neurofibromin protein expression as a surrogate for $N F 1$ gene status in our study.

Germline deleterious NF1 variants were identified in four of the six patients. In two of the four patients with a germline NFl mutation we were able to demonstrate biallelic inactivation of this critical tumor suppressor gene with a second somatic hit to the gene. Of interest, we identified deleterious germline variants in other known familial cancer genes, including a frameshift deletion in FANCC and a splice site mutation in TSCl-both of these occurred in patients who also had germline deleterious variants in NF1. Although this could be chance, it could be that the combination of two germline changes increases the penetrance of the phenotype. This phenomenon has been demonstrated on other hereditary diseases: for example, previous data have demonstrated that patients with the clinical finding of familial pancreatitis often have two distinct germline events that together contribute to the phenotype [29, 30]. In two of the six cases, we did not identify a disease-causing germline $N F 1$ variant, as the remainder of the germline variants identified in the coding region of this gene were synonymous. However, the clinical history of NF1 in each patient in our cohort is well documented, and previous studies have highlighted the difficulties in identifying germline NF1 mutations, as numerous molecular approaches are necessary for comprehensive variant identification [31]. Thus, our whole-exome sequencing approach was likely not sensitive enough to detect all the disease-causing germline alterations in our cohort.

Taken together, our data reveal the importance of somatic NF1 mutation and chromosome 22 loss in duodenal neuroendocrine tumors in patients with NF1, highlighting the unique genomic landscape of this syndromic neoplasm. 
Acknowledgements We acknowledge the following sources of support:NIH/NCI P50 CA62924; NIH/NIDDK K08 DK107781; Sol Goldman Pancreatic Cancer Research Center; Buffone Family Gastrointestinal Cancer Research Fund; Kaya Tuncer Career Development Award in Gastrointestinal Cancer Prevention; AGA-Bernard Lee Schwartz Foundation Research Scholar Award in Pancreatic Cancer; Sidney Kimmel Foundation for Cancer Research Kimmel Scholar Award; AACR-Incyte Corporation Career Development Award for Pancreatic Cancer Research; Rolfe Pancreatic Cancer Foundation; Joseph C Monastra Foundation; The Gerald O Mann Charitable Foundation (Harriet and Allan Wulfstat, Trustees); Lisa Waller Hayes Foundation; Nijbakker Morra Foundation; Dutch Digestive Foundation (CDG 14-02); German Research Foundation (DFG); Associazione Italiana Ricerca Cancro (grant number: 12182); FP7 European Community Grant Cam-Pac (no: 602783); Italian Ministry of University (FIRB RBAP10AHJB); NIH/NCI R00 CA190889.

\section{Compliance with ethical standards}

Conflict of interest LDW is a paid consultant for Personal Genome Diagnostics. The other authors report no conflict of interest.

\section{References}

1. Gutmann DH,Ferner RE,Listernick RH, et al. Neurofibromatosis type 1. Nat Rev Dis Primers. 2017;3:17004

2. Relles D, Baek J, Witkiewicz A, et al. Periampullary and duodenal neoplasms in neurofibromatosis type 1: two cases and an updated 20-year review of the literature yielding 76 cases. J Gastrointest Surg. 2010;14:1052-61.

3. Zhang M, Wang Y, Jones S, et al. Somatic mutations of SUZ12 in malignant peripheral nerve sheath tumors. Nat Genet. 2014;46: 1170-2.

4. Jiao Y, Shi C, Edil BH, et al. DAXX/ATRX, MEN1, and mTOR pathway genes are frequently altered in pancreatic neuroendocrine tumors. Science. 2011;331:1199-203.

5. Francis JM, Kiezun A, Ramos AH, et al. Somatic mutation of CDKN1B in small intestine neuroendocrine tumors. Nat Genet. 2013;45:1483-6.

6. Banck MS, Kanwar R, Kulkarni AA, et al. The genomic landscape of small intestine neuroendocrine tumors. J Clin Invest. 2013;123: 2502-8.

7. Cao Y, Gao Z, Li L, et al. Whole-exome sequencing of insulinoma reveals recurrent T372R mutations in YY1. Nat Commun. 2013;4:2810

8. Maertens O, Prenen H, Debiec-Rychter M, et al. Molecular pathogenesis of multiple gastrointestinal stromal tumors in NF1 patients. Hum Mol Genet. 2006;15:1015-23.

9. Hosoda W, Chianchiano P, Griffin JF, et al. Genetic analyses of isolated high-grade pancreatic intraepithelial neoplasia (HG-PanIN) reveal paucity of alterations in TP53 and SMAD4. J Pathol. 2017;242:16-23.

10. Van Loo P, Nordgard SH, Lingjaerde OC, et al. Allele-specific copy number analysis of tumors. Proc Natl Acad Sci USA. 2010;107:16910-5.

11. Talevich E, Shain AH, Botton T, et al. CNVkit: genome-wide copy number detection and visualization from targeted DNA sequencing. PLoS Comput Biol. 2016;12:e1004873.
12. Li H, Durbin R. Fast and accurate short read alignment with Burrows-Wheeler transform. Bioinformatics. 2009;25:1754-60.

13. Koboldt DC, Zhang Q, Larson DE, et al. VarScan 2: somatic mutation and copy number alteration discovery in cancer by exome sequencing. Genome Res. 2012;22:568-76.

14. Roberts NJ, Norris AL, Petersen GM, et al. Whole genome sequencing defines the genetic heterogeneity of familial pancreatic cancer. Cancer Discov. 2016;6:166-75.

15. Klimstra DS, Arnold R, Capella C, et al. Neuroendocrine neoplasms of the pancreas. In WHO Classification of Tumours of the Digestive System. 4th ed. Lyon: International Agency for Research on Cancer; 2010.

16. Kloppel G, Klimstra DS, Hruban RH, et al. Pancreatic neuroendocrine tumors: update on the new world health organization classification. AJSP: Rev Rep. 2017;22:233-9.

17. Lie JT. Vasculopathies of neurofibromatosis type 1 (von Recklinghausen Disease). Cardiovasc Pathol. 1998;7:97-108.

18. Usui M, Matsuda S, Suzuki H, et al. Somatostatinoma of the papilla of Vater with multiple gastrointestinal stromal tumors in a patient with von Recklinghausen's disease. J Gastroenterol. 2002:37:947-53.

19. Garbrecht N, Anlauf M, Schmitt A, et al. Somatostatin-producing neuroendocrine tumors of the duodenum and pancreas: incidence, types, biological behavior, association with inherited syndromes, and functional activity. Endocr Relat Cancer. 2008;15:229-41.

20. Trofatter JA, MacCollin MM, Rutter JL, et al. A novel moesin-, ezrin-, radixin-like gene is a candidate for the neurofibromatosis 2 tumor suppressor. Cell. 1993;72:791-800.

21. Rouleau GA, Merel P, Lutchman M, et al. Alteration in a new gene encoding a putative membrane-organizing protein causes neuro-fibromatosis type 2. Nature. 1993;363:515-21.

22. Hulsebos TJ, Plomp AS, Wolterman RA, et al. Germline mutation of INI1/SMARCB1 in familial schwannomatosis. Am J Hum Genet. 2007;80:805-10.

23. Sohier P, Luscan A, Lloyd A, et al. Confirmation of mutation landscape of NF1-associated malignant peripheral nerve sheath tumors. Genes Chromosomes Cancer. 2017;56:421-6.

24. Lee W, Teckie S, Wiesner T, et al. PRC2 is recurrently inactivated through EED or SUZ12 loss in malignant peripheral nerve sheath tumors. Nat Genet. 2014;46:1227-32.

25. Pemov A, Li H, Patidar R, et al. The primacy of NF1 loss as the driver of tumorigenesis in neurofibromatosis type 1-associated plexiform neurofibromas. Oncogene. 2017;36:3168-77.

26. Gutmann DH, McLellan MD, Hussain I, et al. Somatic neurofibromatosis type 1 (NF1) inactivation characterizes NF1-associated pilocytic astrocytoma. Genome Res. 2013;23:431-9.

27. Stenman A, Svahn F, Welander J, et al. Immunohistochemical NF1 analysis does not predict NF1 gene mutation status in pheochromocytoma. Endocr Pathol. 2015;26:9-14.

28. Burnichon N, Buffet A, Parfait B, et al. Somatic NF1 inactivation is a frequent event in sporadic pheochromocytoma. Hum Mol Genet. 2012;21:5397-405.

29. LaRusch J, Barmada MM, Solomon S, et al. Whole-exome sequencing identifies multiple, complex etiologies in an idiopathic hereditary pancreatitis kindred. JOP. 2012;13:258-62.

30. Pfutzer RH, Barmada MM, Brunskill AP, et al. SPINK1/PSTI polymorphisms act as disease modifiers in familial and idiopathic chronic pancreatitis. Gastroenterology. 2000;119:615-23.

31. Jett K, Friedman JM. Clinical and genetic aspects of neurofibromatosis 1. Genet Med. 2010;12:1-11. 